www.jmscr.igmpublication.org

Impact Factor (SJIF): 6.379

Index Copernicus Value: 71.58

ISSN (e)-2347-176x ISSN (p) 2455-0450

crossref DOI:_https://dx.doi.org/10.18535/jmscr/v6i4.72

Journal Of Medical Science And Clinical Research

IGM Publication

An Official Publication of IGM Publication

\title{
Surveillance of Antibiotic Resistance pattern of Urinary Tract infections in Pregnant Women - our experience in an Indian teaching hospital
}

\author{
Authors \\ Debasish Sahoo, Kundan Kumar Sahu \\ Department of Microbiology, IMS and SUM hospital, Siksha “O” Anusandhan University, K8, \\ Kalinganagar,Bhubaneswar-751003, Odisha, India \\ *Corresponding Author \\ Dr Kundan Kumar Sahu \\ Associate Professor, Department of Microbiology, IMS and SUM hospital, Siksha "O" Anusandhan \\ University, K8, Kalinganagar, Bhubaneswar-751003, Odisha, India
}

\begin{abstract}
Background: Urinary tract infections (UTIs) are one of the most common bacterial infections in women. During pregnancy physiological changes, like frequency, mimic UTI symptoms and therefore bacteriological cultures are needed to confirm the diagnosis. However, in developing countries antibiotic therapy is commonly initiated without culture confirmation.

Methods: We have screened bacteriuria among pregnant women with and without UTI symptoms in a tertiary care teaching hospital. A total of 1000 urine samples were evaluated with nitrite and leukocyte esterase tests, using urine culture and/or dipslide with species identification as reference.

Results: The prevalence of culture-proven UTI among pregnant women with UTI symptoms was $4 \%$. Since treatment is initiated based only on the presence of symptoms, 96\% were erroneously given antibiotics. Further, there is a high prevalence of resistance to commonly used antibiotics, with $18 \%$ ESBL and $36 \%$ multidrug resistant Escherichia coli strains. With culture morphology and biochemical tests were mostly able to identify E. coli and negative cultures. Mixed Gram-negative flora, suggesting fecal contamination was, however, in the majority of cases interpreted as a single pathogenic bacterium and would have resulted in antibiotic treatment.

Conclusions. To prevent excessive use of antibiotics, dipslide possibly supported by a combination of nitrite and leukocyte esterase tests can be used. Trained frontline health care professionals correctly diagnosed E. coli UTI and negative urine cultures, which would help preventing antibiotic misuse. In addition, regular screening for antibiotic resistance would improve correct treatment.

Keywords: Urinary tract infections; E. coli; antibiotics; pregnant women, India.
\end{abstract}

\section{Introduction}

Urinary tract infections (UTIs) belong to the most common bacterial infections worldwide, primarily affecting women and are a frequent indication for antibiotic therapy ${ }^{[1]}$. In contrast, asymptomatic bacteriuria $(\mathrm{ABU})$, is often benign and does not require antibiotic therapy in non pregnant and otherwise healthy women ${ }^{[2]}$. However, during pregnancy, 25-30\% of pregnant women with $\mathrm{ABU}$ are at risk of developing acute 
pyelonephritis $^{[3]}$, associated with complications such as preterm birth ${ }^{[4]}$. Although screening for and antibiotic treatment of $\mathrm{ABU}$ in pregnant women to reduce the risk of acute pyelonephritis is common in developed countries ${ }^{[5]}$, the beneficial effects of such an intervention have recently been questioned ${ }^{[6]}$. In non pregnant women, management of uncomplicated UTI relies on clinical diagnosis and empiric antibiotic therapy $^{[7]}$. During pregnancy, physiological changes, such as micturation frequency, and during the later part of the pregnancy, also nocturia and suprapubic pain ${ }^{[8]}$, may mimic UTI. Therefore, bacteriological diagnosis is particularly important during pregnancy, especially because antibiotic exposure in utero may have deleterious effects on the fetus ${ }^{[9]}$. However, in countries like Bhubaneswar, with limited diagnostic resources, diagnosis of UTI is based on clinical symptoms. Moreover, midwives hand out antibiotics free of charge to pregnant women with suspect UTI. The extensive and widespread use of antibiotics, dispensed without prescription, is a leading cause of resistance among pathogenic bacteria, thus complicating management of infections in the long run ${ }^{[10]}$. Escherichia coli and other Enterobacteriaceae account for the majority of $\mathrm{UTI}^{[1]}$. We recently reported high antibiotic resistance among $E$. coli isolates from Bhubaneswar compared to those from Sweden ${ }^{[11]}$, further demonstrating this relation. The increasing antibiotic resistance calls for deliberate actions to change the current treatment policy. We speculate that a high proportion of pregnant women with lower UTI symptoms receive antibiotics without having an infection. The limited diagnostic services available in Bhubaneswar and other lowincome countries, leading to overconsumption of antibiotics, is likely an important reason promoting the high prevalence of antibiotic resistance. We here investigate the prevalence of bacteriuria in pregnant women with and without symptoms consistent with lower UTI and the antibiotic susceptibility of $E$. coli isolates in relation to the empirically prescribed antibiotics in
Bhubaneswar. Further, the reliability of nitrate and leukocyte esterase tests, as well as microscopy for identification of bacteriuria in pregnant women, and lastly, the possibility to use point of care dipslide for urine culture in areas without clinical laboratories were investigated.

\section{Materials and Methods}

A total of 395 urine samples from pregnant women with or without having symptoms of UTI were collected during October 2014 to January 2016. The age of people included in the study ranged from 25-40 years. Verbal informed consent from target population and approval from institutional research ethical committee were obtained before starting the experiment.

Female patients aged between 25-40 years with uncomplicated UTI symptoms like frequency, urgency and dysuria were included in the study. Pregnant women on antibiotics within the last 2 weeks and those who could not give consent to participate in the study were excluded. Clean catch midstream urine samples were collected into a sterile screw capped universal container by standard method. The samples were labeled and $0.2 \mathrm{mg}$ of boric acid was added to prevent the bacterial growth in urine samples. The samples were cultured on cysteine-lactose electrolyte deficient agar and blood agar using a sterile $4 \mathrm{~mm}$ platinum wired calibrated loop for the isolation of microorganisms. The plates were incubated for overnight at $37{ }^{\circ} \mathrm{C}$ and the samples were considered positive when an organism was cultured at a concentration of $10^{4} \mathrm{CFU} / \mathrm{mL}$ which was estimated through multiplying the isolated colonies by 1000 . The isolates were identified up to the species level by standard biochemical tests $^{[15]}$.

Antibiotic sensitivity testing was performed by the modified disc diffusion method as per the recommendations ${ }^{[16]}$. Inoculums adjusted to 0.5 McFarland standard was swabbed on Mueller Hinton agar plates for antibiotic sensitivity assay. Eight groups of antimicrobials such as penicillins, cephalosporins, fluoroquinolones, aminoglyco- 
sides, macrolides, lincosamides, glycopeptides and sulfonamides were selected based on frequent prescription and used in this study. Among the group, the antibiotics tested were amoxicillin (10 $\mu \mathrm{g})$, oxacillin $(10 \mu \mathrm{g})$, cloxacillin $(5 \mu \mathrm{g})$, cefotaxime $(10 \mu \mathrm{g})$, ceftriaxone $(30 \mu \mathrm{g})$, nalidixic acid $(30 \mu \mathrm{g})$, ciprofloxacin $(5 \mu \mathrm{g})$, norfloxacin $(10$ $\mu \mathrm{g})$, amikacin $(30 \mu \mathrm{g})$, gentamycin $(10 \mu \mathrm{g})$, erythromycin $(10 \mu \mathrm{g})$, clindamycin $(2 \mu \mathrm{g})$, vancomycin $(30 \mu \mathrm{g})$ and co-trimoxazole $(30 \mu \mathrm{g})$. Statistical analysis was done using Chi-square test and student's t-test.

\section{Results}

Among the 1000 samples collected, 180 were laboratory confirmed cases of UTI with a positive culture percentage of $46.6 \%$ (Table 1). The age of the pregnant women ranged from 25-40 years. Majority (50.6\%) of the study participants were in the age group of 30-34 years. The highest UTI were observed at third trimester gestational age $(\mathrm{n}=103 ; 57.2 \%)$.

From the 180 isolates, 154 were Gram negative while 26 were Gram positive bacteria (Figure 1). Escherichia coli (E. coli) was the most common organism isolated accounting for 79 (43.9\%) and the second highest organism was Klebsiella oxytoca $(K$. oxytoca) $(n=35 ; 19.4 \%)$ followed by Klebsiella pneumoniae (K. pneumoniae) $(n=24$; $13.3 \%$ ). The other bacterial isolates obtained in the study were Enterococcus faecalis (E. faecalis), Staphylococcus saprophyticus (S. saprophyticus), Staphylococcus aureus (S. aureus), Proteus mirabilis (P. mirabilis), Proteus vulgaris (P. vulgaris), Pseudomonas aeruginosa (P. aeruginosa), Citrobacter koseri (C. koseri) and Citrobacter amalonaticus (C. amalonaticus).

The frequency of occurrence of other bacterial isolates were $\mathrm{E}$. faecalis $(\mathrm{n}=12 ; 6.7 \%), \mathrm{S}$. saprophyticus $(\mathrm{n}=10 ; 5.5 \%), \mathrm{P}$. mirabilis $(\mathrm{n}=9$; $5.0 \%)$, S. aureus $(n=4 ; 2.2 \%), P$. aeruginosa $(n=3$; $1.7 \%)$, P. vulgaris $(\mathrm{n}=2 ; 1.1 \%)$, C. koseri $(\mathrm{n}=1$; $0.6 \%)$ and C. amalonaticus $(\mathrm{n}=1 ; 0.6 \%)$. Antibiotic sensitivity pattern of the bacterial isolates revealed that $\mathrm{E}$. coli with $89.9 \%$ sensitivity to amikacin, $83.5 \%$ to co-trimoxazole, $79.7 \%$ to clindamycin and $77.2 \%$ to ciprofloxacin (Table 2). The antibiotics which had poor activity against E. coli were cloxacillin (13.9\%), gentamycin (12.6\%), vancomycin (8.9\%), erythromycin (7.6\%), amoxicillin $(5.1 \%)$ and oxacillin (2.5\%). K. oxytoca showed $88.5 \%$ sensitivity to ciprofloxacin and $62.8 \%$ to nalidixic acid while sensitivity to erythromycin was $0 \%$. Most of the antibiotics were effective against $\mathrm{K}$. pneumoniae with co-trimoxazole has significant activity $(91.6 \%)$ followed by cefotaxime $(83.3 \%)$. Gross $100 \%$ sensitivity was noted on nalidixic acid against E. faecalis and P. mirabilis. S. saprophyticus was $90 \%$ sensitive to cotrimoxazole and $80 \%$ to nalidixic acid, clindamycin and vancomycin. A complete $100 \%$ sensitivity to cefotaxime and clindamycin was observed with P. aeruginosa, P. vulgaris, C. koseri and $\mathrm{C}$. amalonaticus. The isolated uropathogens revealed the presence of high levels of single and multiple antimicrobial resistances against commonly prescribed drugs as shown in Table 3. Amikacin had the highest overall sensitivity $(n=138 ; 76.7 \%)$ against the 180 isolates tested. This was followed by ciprofloxacin $(n=132$; $73.3 \%)$ and clindamycin $(n=124 ; 68.9 \%)$. Cefotaxime and nalidixic acid were exhibited $65.0 \%$ and $63.9 \%$ sensitivity against the isolates. Ceftriaxone had the overall sensitivity of $52.8 \%$. The other antibiotics were recorded lesser than $50 \%$ of the sensitivity as follows: co-trimoxazole $45.0 \%$, cloxacillin $31.6 \%$, oxacillin $31.1 \%$, norfloxacin $29.4 \%$, vancomycin $28.9 \%$, amoxicillin. 
Table 1 Characteristics of UTI in pregnant women.

\begin{tabular}{|l|c|c|c||}
\hline & & \multicolumn{2}{||}{} \\
\hline Variables & & \multicolumn{2}{|c||}{ Numbers (n=180) Percentage } \\
\hline Age & $25-29$ years & 87 & 48.3 \\
\hline & $30-34$ years & 91 & 50.6 \\
\hline & $\geq$ & & \\
\hline \multirow{3}{*}{ Gestational period } & 35 years & 2 & 1.1 \\
\hline & First trimester & 21 & 11.7 \\
\hline & Second trimester & 56 & 31.1 \\
\hline & Third trimester & 103 & 57.2 \\
\hline
\end{tabular}

Table 2 Antibiotic sensitivity pattern of urinary isolates. $\mathrm{n}(\%)$.

\begin{tabular}{|c|c|c|c|c|c|c|c|c|c|c|c|c|}
\hline Antibiotic & E. coli & K. oxytoca & $\mathrm{K}$. & & aecalis & S. & P. mirabilis & S. aureus & P. & P. vulgaris & C. koseri & C. \\
\hline & & & pneumoniae & & & saprophyti & & & eruginosa & & & amalonticus \\
\hline illin $(10 \mu \mathrm{g})$ & $4(5.1)$ & 11 & $8(33.3)$ & 9 & $(75.0)$ & $7(70.0)$ & 7) & $(50.0)$ & $2(66.6)$ & $\begin{array}{c}0 \\
(0.0) \\
\end{array}$ & $0(0.0)$ & $1(100.0)$ \\
\hline$(10 \mu \mathrm{g})$ & $2(2.5)$ & 12 & $16(66.7)$ & 9 & $(75.0)$ & $7(70.0)$ & $6(66.6)$ & $1(25.0)$ & $2(66.6)$ & $\begin{array}{c}0 \\
(0.0)\end{array}$ & $0(0.0)$ & $1(100.0)$ \\
\hline Clox & $11(13.9)$ & $8(23.0)$ & $10(41.6)$ & & (91.6) & $3(30.0)$ & $8(8$ & $3(75.0)$ & $1(33.3)$ & $\begin{array}{c}1 \\
(50.0)\end{array}$ & $0(0.0)$ & $1(100.0)$ \\
\hline Cefotaxime $(10 \mu \mathrm{g})$ & $53(67.0)$ & $17(48.5)$ & $20(83.3)$ & 7 & $(58.3)$ & $4(40.0)$ & $8(88.9)$ & $1(25.0)$ & $\begin{array}{c}3 \\
(100.0) \\
\end{array}$ & $\begin{array}{c}2 \\
(100.0) \\
\end{array}$ & $\begin{array}{c}1 \\
(100.0) \\
\end{array}$ & $1(100.0)$ \\
\hline Ceft & $47(59.5)$ & & $15(62$ & 7 & $(58.3)$ & $6(60.0)$ & & $0.0)$ & $2(66.6)$ & $\begin{array}{c}1 \\
(50.0) \\
\end{array}$ & $\begin{array}{c}1 \\
(100.0) \\
\end{array}$ & $0(0.0)$ \\
\hline $\begin{array}{l}\text { Nalidixic acid }(30 \\
\mu \mathrm{g})\end{array}$ & $38(48.1)$ & $22(62.8)$ & $17(70.8)$ & 12 & $(100.0)$ & $8(80.0)$ & $9(100.0)$ & $3(75.0)$ & $2(66.6)$ & $\begin{array}{c}2 \\
(100.0) \\
\end{array}$ & $\begin{array}{c}1 \\
(100.0) \\
\end{array}$ & $1(100.0)$ \\
\hline$\mu \mathrm{g})$ & $61(77.2)$ & & $11(45.8)$ & 8 & $(66.6)$ & $6(60.0)$ & $8(88.9)$ & $2(50.0)$ & $\begin{array}{c}3 \\
(100.0) \\
\end{array}$ & $1(50.0)$ & $\begin{array}{c}1 \\
(100.0) \\
\end{array}$ & $0(0.0)$ \\
\hline Norfloxacin $(10 \mu \mathrm{g})$ & $45(56.9)$ & $10(28.6)$ & $14(58.3)$ & 9 & $(75.0)$ & $4(40.0)$ & 6.6) & $1(25.0)$ & $2(66.6)$ & $1(50.0)$ & $\begin{array}{c}1 \\
(100.0) \\
\end{array}$ & $1(100.0)$ \\
\hline Amikacin $(30 \mu \mathrm{g})$ & $71(89.9)$ & $18(51.4)$ & $19(79.1)$ & 3 & $(75.0)$ & $5(50.0)$ & $8(88.9)$ & $3(75.0)$ & $2(66.6)$ & $2(100.0)$ & $0(0.0)$ & $1(100.0)$ \\
\hline Gentamycin $(10 \mu \mathrm{g})$ & $10(12.6)$ & $5(14.2)$ & $17(70.8)$ & 4 & (33.3) & $5(50.0)$ & $3(33.3)$ & $2(50.0)$ & $2(66.6)$ & $1(50.0)$ & $0(0.0)$ & $0(0.0)$ \\
\hline Erythromycin $(10 \mu \mathrm{g})$ & $6(7.6)$ & $0(0.0)$ & $(29.1)$ & $J$ & (41.6) & $7(70.0)$ & 4.4) & $5.0)$ & $0(0.0)$ & $1(50.0)$ & $0(0.0)$ & $0(0.0)$ \\
\hline Clindamycin $(2 \mu \mathrm{g})$ & $63(79.7)$ & $18(51.4)$ & $10(41.6)$ & 9 & $(75.0)$ & $8(80.0)$ & $6(66.6)$ & $3(75.0)$ & \begin{tabular}{|c|}
3 \\
$(100.0)$ \\
\end{tabular} & $2(100.0)$ & $\begin{array}{c}1 \\
(100.0) \\
\end{array}$ & $1(100.0)$ \\
\hline Vancomycin $(30 \mu \mathrm{g})$ & $7(8.9)$ & $(56.0)$ & $18(75.0)$ & 8 & $(66.6)$ & $8(80.0)$ & $2.2)$ & $4(100.0)$ & $1(33.3)$ & $1(50.0)$ & $0(0.0)$ & $0(0.0)$ \\
\hline o-trimoxazole $(30 \mu \mathrm{g})$ & $66(83.5)$ & $20(57.1)$ & $22(91.6)$ & & (83.3) & $9(90.0)$ & $6(66.6)$ & $3(75.0)$ & \begin{tabular}{|c|}
3 \\
$(100.0)$ \\
\end{tabular} & $2(100.0)$ & $0(0.0)$ & $0(0.0)$ \\
\hline
\end{tabular}

Table 3 Antibiotic sensitivity pattern of the isolates

\begin{tabular}{|c|c|c|c|c|}
\hline \multirow[t]{2}{*}{ Antibiotics } & & \multirow[t]{2}{*}{ Frequency } & \multicolumn{2}{|c|}{ Percentages } \\
\hline & & & Sensitive & Resistance \\
\hline \multirow[t]{2}{*}{ Penicillin } & Amoxicillin & 51 & 28.3 & 71.7 \\
\hline & Oxacillin & 56 & 31.1 & 68.9 \\
\hline & \multirow[b]{2}{*}{ Cloxacillin } & 57 & 316 & 684 \\
\hline \multirow[b]{2}{*}{ Cephalosporin } & & \multirow[b]{2}{*}{117} & & \\
\hline & Cefotaxime & & 65.0 & 35.0 \\
\hline \multirow{3}{*}{ Fluoroquinolones } & Ceftriaxone & 95 & 52.8 & 47.2 \\
\hline & Nalidixic acid & 115 & 63.9 & 36.1 \\
\hline & Ciprofloxacin & 132 & 73.3 & 26.7 \\
\hline \multirow[b]{2}{*}{ Aminoglycosides } & Norfloxacin & 53 & 29.4 & 70.6 \\
\hline & Amikacin & 138 & 76.7 & 23.3 \\
\hline \multirow[b]{2}{*}{ Macrolide } & Gentamycin & 49 & 27.2 & 72.8 \\
\hline & Erythromycin & 33 & 18.3 & 81.7 \\
\hline Lincosamides & Clindamycin & 124 & 68.9 & 31.1 \\
\hline Glycopeptide & Vancomycin & 52 & 28.9 & 71.1 \\
\hline Sulfonamide & Co-trimoxazole & 81 & 45.0 & 55.0 \\
\hline
\end{tabular}




\section{Discussion}

Clinical diagnosis and empiric antibiotic therapy of UTI among pregnant women lead to massive antibiotic misuse, inadvertently rendering the first line of drugs ineffective. Diagnostic tests, easy to carry out and evaluate also by inexperienced staff, are therefore necessary. In this study, we investigated a total of 2562 patients for bacteriuria. Of these 1621 were urine samples from pregnant women in IMS and SUM Hospital, Bhubaneswar with and without UTI symptoms, in order to verify the prevalence of cystitis and $A B U$. Because physiological changes during pregnancy might cause symptoms similar to cystitis, a low frequency of bacteriologically confirmed UTI among patients with symptoms was anticipated. Although the prevalence of UTI among pregnant women in our study is close to those reported regionally ${ }^{[17,18]}$, it is particularly alarming that our observations show that as many as $96 \%$ of patients are routinely treated with antibiotic therapy that they do not require. The positive correlation between antibiotic misuse and development of drug resistance ${ }^{[19]}$ may in part explain the high antibiotic resistance seen in developing countries as we and others have demonstrated $^{[11,20]}$. To improve the diagnosis, we evaluated nitrite and leukocyte esterase tests and a simplified culture method, dipslide. In line with previous reports, nitrite and leukocyte esterase tests alone demonstrated insufficient diagnostic value in this study. The combined results of these 2 tests demonstrated acceptable specificity and low sensitivity but were still of limited value. Although the nitrite test had high specificity, the sensitivity was low, especially among pregnant women, which is line with other studies ${ }^{[21]}$ and may relate to frequent urination leading to reduced bacterial concentration. Further, presence of vitamin $\mathrm{C}$ in urine is another confounding factor $^{[22]}$, which cannot be ruled out among our patients. During pregnancy, the specificity of urinary leukocytes as an indicator for bacteriuria per se might be questionable because of elevated urine specific gravity that may give false negative results $^{[22]}$. Similarly, low sensitivity was obtained in urine samples collected from non pregnant women. Moreover, false negative results have been reported with common antibiotics like cephalosporins $^{[23]}$. Also, the poor correlation between enzymatic activity and microscopic detection of leukocytes in urine is a major concern. Even though microscopy is regarded as the golden standard for this analysis, high variability between replicate leukocyte counts suggest that the analysis suffers substantial draw backs, when performed in the clinical setting. Moreover, false negative results might be due to cell lyses if analysis is delayed. In addition, microscopic analyses of leukocyturia are time consuming if carried out with care. Taken together, detection of leukocytes especially using microscopy but also leukocyte esterase test are poor markers for the detection of UTI particularly among pregnant women and is in line with previous studies $^{[24,25]}$. The use of dipslide culture was, on the other hand, particularly rewarding in urine samples with no bacterial growth. These samples constitute the majority and were correctly diagnosed. Therefore, even in the hands of inexperienced healthcare providers this would substantially decrease the antibiotic use. The identification of E. coli and other Enterobacteriaceae was also satisfactorily evaluated with the dipslide test even with inexperienced medical doctors and nurses. This can in part be attributed to the E. coli-specific agar containing $\beta$ glucuronidase, resulting in a color change. However, false negative results can occur in rare cases if the strain lacks the enzyme ${ }^{[13]}$. Also, false positive results may occur among Citrobacter spp. ${ }^{[26]}$. Interestingly, we demonstrated that nurses with very limited training, and with no access to a professional microbiological laboratory or expert personnel, correctly diagnosed E. coli UTI in almost $80 \%$ of cases. To accurately define mixed fecal flora using dipslide ${ }^{[27]}$ was problematic, and the nurses prescribed antibiotics in $91 \%$ of such cases. In the current study, contamination was observed in 5\%, whereas others have 
demonstrated up to $10 \%{ }^{[28]}$, which would implicate risk of overinterpretation. Another drawback for dipslide culture especially among pregnant women is the failure to detect GBS, which is in line with observations made employing 2-medium dipslides ${ }^{[29]}$. Treatment recommendations differ between countries. In IMS and SUM Hospital, Bhubaneswar, pregnant women with UTI receive amoxicillin, amoxicillin/clavulanic acid, or cephalexin without laboratory verification and susceptibility testing, whereas nitrofurantoin or amoxicillin are recommended in Kenya, Tanzania, and Rwanda ${ }^{[30-32]}$. The use of nitrofurantoin and pivmecillinam are also recommended by international guidelines ${ }^{[33]}$. In our study, E. coli was highly resistant to many of the drugs used for treatment of pregnant women like $66 \%$ resistant to ampicillin (amoxacillin), 38\% to amoxicillin/ clavulanic acid, and $18 \%$ to cephalexin, which decreases the treatment options. In addition, we report a high ESBL prevalence (18\%), even higher than the general ESBL prevalence in Africa $^{[34]}$. As a consequence, almost all pregnant women presenting with UTI symptoms will receive antibiotics either not indicated at all or with high risk of being ineffective. International and East African regional guidelines recommend nitrofurantoin and during the last 2 trimesters also pivmecillinam. An appropriate alternative would therefore be nitrofurantoin or pivmecillinam, where low resistance is observed ${ }^{[30,32,33]}$.

The high antibiotic consumption proves the importance of continuously reviewing the susceptibility pattern of uropathogens. It also calls for diagnostic methods possible to use in clinics with no access to laboratory service. To the best of our knowledge, this is the first study investigating the practicability of the dipslide culture test under clinically relevant conditions in a low-resource country. To introduce dipslide in clinical routine, possibly supported with nitrite and leukocyte esterase tests would substantially reduce the misuse of antibiotics. Healthcare professionals can learn to correctly evaluate samples with no bacterial growth. Compared to clinical diagnosis alone, the use of dipslide would imply substantially decreased antibiotic consumption and as a consequence decreased risk for development of resistance among bacterial pathogens. The massive amount of saved antibiotics might furthermore outweigh, at least in part, the costs associated with the increased diagnostic efforts.

\section{Acknowledgement}

Authors are grateful to President, Siksha 'O' Anusandhan University, Bhubaneswar for extended facility in research.

\section{References}

1. Dielubanza EJ, Schaeffer AJ. Urinary tract infections in women. Med Clin North Am 2011; 95:27-41.

2. Nicolle LE. Asymptomatic bacteriuria: review and discussion of the IDSA guidelines. Int J Antimicrob Agents 2006; 28 Suppl 1:S42-8.

3. Sheffield JS, Cunningham FG. Urinary tract infection in women. Obstet Gynecol 2005; 106:1085-92.

4. Mazor-Dray E, Levy A, Schlaeffer F, Sheiner E. Maternal urinary tract infection: is it independently associated with adverse pregnancy outcome? J Matern Fetal Neonatal Med 2009; 22:124-8.

5. Smaill F, Vazquez JC. Antibiotics for asymptomatic bacteriuria in pregnancy. Cochrane Database of Systematic Reviews 2007.

6. Kazemier BM, Koningstein FN, Schneeberger $\mathrm{C}$, et al. Maternal and neonatal consequences of treated and untreated asymptomatic bacteriuria in pregnancy: a prospective cohort study with an embedded randomised controlled trial. Lancet Infect Dis 2015; 15:1324-33.

7. Gupta K, Hooton TM, Naber KG, et al. International clinical practice guidelines for the treatment of acute uncomplicated 
cystitis and pyelonephritis in women: a 2010 update by the infectious diseases society of America and the European Society for Microbiology and Infectious Diseases. Clin Infect Dis 2011; 52: E103E20.

8. FitzGerald MP, Graziano S. Anatomic and functional changes of the lower urinary tract during pregnancy. Urol Clin North Am 2007; 34:7-12.

9. Kenyon S PK, Jones DR, Brocklehurst P, Marlow N, Salt A, Taylor DJ Childhood outcomes after prescription of antibiotics to pregnant women with preterm rupture of the membranes: 7-year follow-up of the ORACLE I trial. Lancet 2008; 372: 131018.

10. Levy SB, Marshall B. Antibacterial resistance worldwide: causes, challenges and responses. Nat Med 2004; 10:S122-9.

11. Ramos NL, Sekikubo M, Dzung DT, et al. Uropathogenic Escherichia coli isolates from pregnant women in different countries. J Clin Microbiol 2012; 50:3569-74.

12. Hooton TM, Roberts PL, Cox ME, Stapleton AE. Voided midstream urine culture and acute cystitis in premenopausal women. N Engl J Med 2013; 369:188391.

13. Dalet F, Segovia T. Evaluation of a new agar in Uricult-Trio for rapid detection of Escherichia coli in urine. J Clin Microbiol 1995; 33:1395-8.

14. Livermore DM. Defining an extendedspectrum beta-lactamase. Clin Microbiol Infect 2008; 14(Suppl 1):3-10.

15. Magiorakos AP, Srinivasan A, Carey RB, et al. Multidrug-resistant, extensively drug-resistant and pandrug-resistant bacteria: an international expert proposal for interim standard definitions for acquired resistance. Clin Microbiol Infect 2012; 18:268-81.
16. Holten KB, Onusko EM. Appropriate prescribing of oral beta-lactam antibiotics. Am Fam Physician 2000; 62:611-20.

17. Hamdan HZ, Ziad AH, Ali SK, Adam I. Epidemiology of urinary tract infections and antibiotics sensitivity among pregnant women at Khartoum North Hospital. Ann Clin Microbiol Antimicrob 2011; 10:2.

18. Alemu A, Moges F, Shiferaw Y, et al. Bacterial profile and drug susceptibility pattern of urinary tract infection in pregnant women at University of Gondar Teaching Hospital, Northwest Ethiopia. BMC Res Notes 2012; 5:19.

19. Goossens H, Ferech M, Vander Stichele R, Elseviers M; ESAC Project Group. Outpatient antibiotic use in Europe and association with resistance: a crossnational database study. Lancet 2005; 365:579-87.

20. Del Arco A, Tortajada B, de la Torre J, et al. The impact of an antimicrobial stewardship programme on the use of antimicrobials and the evolution of drug resistance. Eur J Clin Microbiol Infect Dis 2015; 34:247-51.

21. Demilie T, Beyene G, Melaku S, Tsegaye W. Diagnostic accuracy of rapid urine dipstick test to predict urinary tract infection among pregnant women in Felege Hiwot Referral Hospital, Bahir Dar, North West Ethiopia. BMC Res Notes 2014; 7:481.

22. Simerville JA, Maxted WC, Pahira JJ. Urinalysis: a comprehensive review. Am Fam Physician 2005; 71:1153-62.

23. Beer JH, Vogt A, Neftel K, Cottagnoud P. False positive results for leucocytes in urine dipstick test with common antibiotics. BMJ 1996; 313:25.

24. Kupelian AS, Horsley H, Khasriya R, et al. Discrediting microscopic pyuria and leucocyte esterase as diagnostic surrogates for infection in patients with lower urinary tract symptoms: results from a clinical and 
laboratory evaluation. BJU Int 2013; 112:231-8.

25. Leman P. Validity of urinalysis and microscopy for detecting urinary tract infection in the emergency department. Eur J Emerg Med 2002; 9:141-7.

26. Perez JL, Berrocal CI, Berrocal L. Evaluation of a commercial betaglucuronidase test for the rapid and economical identification of Escherichia coli. J Appl Bacteriol 1986; 61:541-5.

27. Schneeberger C, van den Heuvel ER, Erwich JJ, Stolk RP, Visser CE, Geerlings SE. Contamination rates of three urinesampling methods to assess bacteriuria in pregnant women. Obstet Gynecol 2013; 121:299-305.

28. Palmqvist E, Aspevall O, Burman E, Nordin G, Svahn A, Forsum U. Difficulties for primary health care staff in interpreting bacterial findings on a device for simplified urinary culture. Scand J Clin Lab Invest 2008; 68:312-6.

29. Mignini L, Carroli G, Abalos E, Widmer M, Amigot S, Nardin JM, Giordano D, Merialdi M, Arciero G, Del Carmen Hourquescos M; World Health Organization Asymptomatic Bacteriuria Trial Group. Accuracy of diagnostic tests to detect asymptomatic bacteriuria during pregnancy. Obstet Gynecol 2009; 113:346-52.

30. Director Health Services K. Clinical guidelines for the diagnosis and treatment of common conditions in Kenya. 2002: 18.2.3.

31. Ministry of Health and Social Welfare TURoT. Standard Treatment Guidelines and Essential Medicines List. 2013: 85.

32. Ministry of Health Kigali R. Gynecology and Obstetrics Clinical protocols and treatment guidelines. 2012: 71-4.
33. Wagenlehner FM, Schmiemann G, Hoyme $\mathrm{U}$, et al. National S3 guideline on uncomplicated urinary tract infection: recommendations for treatment and management of uncomplicated community-acquired bacterial urinary tract infections in adult patients. Urologe A 2011; 50:153-69.

34. Tansarli GS, Poulikakos P, Kapaskelis A, Falagas ME. Proportion of extendedspectrum $\beta$-lactamase (ESBL)-producing isolates among Enterobacteriaceae in Africa: evaluation of the evidencesystematic review. J Antimicrob Chemother 2014; 69:1177-84. 